\title{
TIMEKEEPING
}

\section{Light-insensitive optical clock}

\section{Thomas Udem}

\section{Interactions between trapped neutral atoms have prevented their use as the ultimate frequency standard in optical clocks. A clever trapping scheme circumvents this problem and may push timekeeping to new limits.}

Almost all clocks consist of two essential parts: an oscillator with periodically occurring events, and a counter to keep track of those events. For example sundials, the oldest known clocks, use Earth's rotation as the oscillator and a human as the counter. Pendulum clocks, first made by Christiaan Huygens in 1656 , have a pendulum oscillator and an automatic mechanical counter to display time. Coming right up to date, on page 321 of this issue, Takamoto and co-workers ${ }^{1}$ demonstrate the latest innovation in timekeeping.

Modern clocks use fast oscillators and electronic counters. In the quartz clocks developed in the 1920s, a small crystal is excited to vibrate typically some 10,000 times per second. In atomic clocks, the nucleus of an atom precesses in the field created by its electrons. Caesium atomic clocks use a standard detection method to follow these precessions ${ }^{2}$ and to feed the resulting oscillatory signal to an electronic counter, which advances the second hand of the clock precisely every 9,192,631,770 cycles. This rather odd value corresponds to the transition frequency between two energy levels of the ground state of the caesium-133 atom and has been defined as the SI unit of time since 1967. Modern caesium atomic clocks can be as accurate as one part in $10^{15}$, which means that time is by far the most precisely measurable physical quantity.

Because clock instability (the variation in its 'ticking', or displayed time flow) can be reduced by increasing the oscillator frequency, it is clear how even today's most precise caesium atomic clocks may be improved: replace the caesium by an optical oscillator. The typical frequencies for such optical oscillators are some 100 or even $1,000 \mathrm{THz}\left(10^{14}\right.$ or
$10^{15}$ cycles per second), and the oscillations are emitted (or absorbed) in the form of light. For a long time, this kind of oscillator was much easier to create than a correspondingly fast optical counter or clock mechanism. Efforts to build a counter that was fast enough began in the early 1960s, but it was only in 1995 that counters were developed that could reach the frequency of visible light ${ }^{3}$. However, these delicate and complicated counters required a number of lasers, filling several large laboratories, and so were not generally considered a serious option for a clock mechanism.

Things changed in 1999 with the introduction of an optical counter based on a pulsed femtosecond $\left(10^{-15}\right.$-second) laser ${ }^{4}$. This compact device can operate continuously for days, so that counting optical oscillations became a simple task, and the first experimental optical clocks appeared $^{5}$. A good optical oscillator requires a sharp transition that gives a strong signal with little noise and a lack of sensitivity to external perturbations. Over the years, the quest for the most suitable oscillator has been pursued along two main lines.

In the first approach, a single ion is trapped in electric fields and brought to rest by laser cooling. If both trap and ion are suitably chosen, the trapping fields will not seriously perturb the clock transition, which is triggered by a highly stable clock laser whose frequency is measured by an optical counter. Various ions are under consideration, and have produced impressive results (see for example ref. 6). But although trapped ions are largely immune to constant but unknown shifts in frequency within the system (so-called systematic uncertainties), they do not give stable enough measurements, because the signal is generated

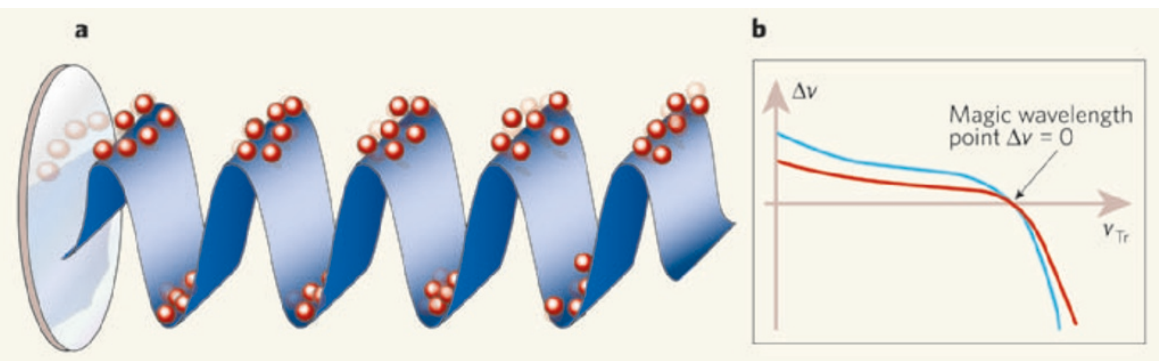

Figure 1 Details of the optical oscillator proposed by Takamoto et al. ${ }^{1}$. a, Strontium atoms (red) are trapped at the crests of a standing wave formed by a laser (blue), which is reflected back onto itself by a mirror. A second laser beam (not shown) measures the clock transition. $\mathbf{b}$, By choosing the appropriate trapping-laser frequency $\nu_{\mathrm{Tr}}$, the perturbation $\Delta v$ of the clock transition can be eliminated. The graph shows $\Delta v$ as a function of $\nu_{\mathrm{Tr}}$ high (blue) and low (red) trapping-laser intensity. At the 'magic wavelength', the clock-transition perturbation vanishes, irrespective of the trapping-laser intensity, which is hard to control with the required accuracy.

from only one ion and must be averaged over a long period. And it has not been possible to trap large numbers of ions without introducing large frequency shifts.

The second approach uses clouds of millions of neutral atoms to produce a much stronger signal, resulting in excellent stability. However, collisions between the atoms result in uncontrollable shifts in frequency. In other words, single-ion standards have high statistical but low systematic uncertainties whereas neutral-atom standards have excellent statistics but poor systematic properties.

Takamoto et al. ${ }^{1}$ demonstrate a method that combines the advantages of both atomic standards. Clouds of neutral strontium atoms are held in a different kind of trap formed at the crests (anti-nodes) of a standing wave produced by a laser (Fig. 1a). In previous attempts, the laser intensity that is needed to keep neutral atoms in place significantly perturbed the transition frequency. Measuring the intensity of the trapping laser to compensate for this negative effect does not work because intensity, unlike frequency or time, cannot be measured sufficiently accurately. Instead, the authors adjust the trapping-laser frequency — which would otherwise be unimportant - so that it shifts the upper and lower energy levels of the clock transition by exactly the same amount, leaving the clock-transition frequency unaltered (Fig. 1b). The frequency of the trapping laser is then set to the correct value - to within $1 \mathrm{MHz}$, which is sufficient to suppress its effect on the clock transition to less than $0.001 \mathrm{~Hz}$ at a clock frequency of $429 \mathrm{THz}$.

All-optical clocks are predicted to keep time within a fractional uncertainty of $10^{-18}$. Such clocks could allow fundamental physical theories to be verified at a new level. Testing general relativity and quantum electrodynamics, and detecting slow variations in the fundamental constants predicted by some theories, rely on the most precise clocks. At the $10^{-18}$ level of accuracy, the gravitational redshift of two clocks differing in their distance from Earth's centre by only $1 \mathrm{~cm}$ would become measurable. Moreover, the optical equivalent of very-long-baseline interferometry may become feasible, allowing higher-resolution astronomical images to be produced by combining data collected by widely separated telescopes. These clocks may also help to improve technologies, such as satellite navigation and broadband network synchronization, that are based on precision timing.

Thomas Udem is at the Max-Planck-Institut für Quantenoptik, Hans-Kopfermann-Strasse 1, 85748 Garching, Germany. e-mail: thomas.udem@mpq.mpg.de

\footnotetext{
1. Takamoto, M., Hong, F.-L., Higashi, R. \& Katori, H. Nature 435, 321-324 (2005).

2. Ramsey, N. F. Phys. Rev. 78, 695-699 (1950).

3. Schnatz, H. et al. Phys. Rev. Lett. 76, 18-21 (1996).

4. Udem, Th. et al. Nature 416, 233-237 (2002)

5. Diddams, S. A. et al. Science 293, 825-828 (2001).

6. Margolis, H. S. et al. Science 306, 1355-1358 (2004).
} 